\title{
Die Crew ist an Bord, die SAQM ist auf Kurs
}

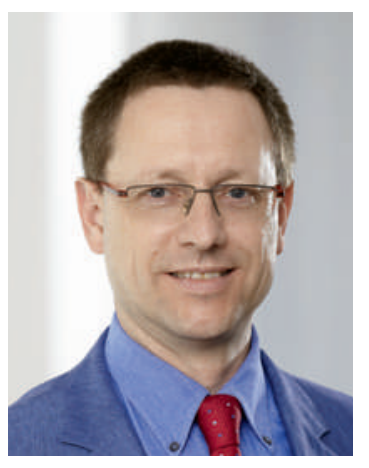

Mit viel Erwartungen haben wir seitens Ressort Daten, Demographie und Qualität DDQ dem 23. Mai 2013 entgegengefiebert, dem ersten Treffen des «Forum Qualität» unserer im vergangenen November gegründeten Schweizerischen Akademie für Qualität in der Medizin SAQM der FMH. Das Treffen des «Dialog Qualität», also der Partnerorganisationen aus dem Gesundheitswesen, hat bereits diesen März stattgefunden und aufgrund der Aussensicht der eingeladenen Organisationen viele interessante und wertvolle Anregungen eingebracht.

Nun also treffen sie ein, die rund 50 Qualitäts-Delegierten unserer Fachgesellschaften, kantonalen Ärztegesellschaften, Dachverbände sowie des VSAO und des VLSS. Das Programm des ersten «Forum Qualität» ist reichbefrachtet und von der Wahl der vier Mitglieder des Steuerungsausschusses der SAQM, welcher die Geschäfte in 2-monatlichem Sit-

\section{Der 6-köpfige Steuerungsausschuss der SAQM bildet die Breite der Ärzte- schaft ab.}

zungs-Rhythmus leiten wird, geprägt. Ex officio im 6-köpfigen Gremium befinden sich bereits der Ressort-Verantwortliche DDQ aus dem FMH-Zentralvorstand sowie die Abteilungsleiterin DDQ. Die breite Palette der Kandidatinnen und Kandidaten ist Spiegelbild des grossen Engagements der Verbände und auch einzelner Kolleginnen und Kollegen. Beides ist unabdingbare Grundlage dafür, dass unsere SAQM zu dem werden kann, was wir uns zum Ziel gesetzt haben: eine lebhaft genutzte Qualitätsorganisation der Ärzteschaft, welche uns gemeinsam in der Realisierung unserer Qualitäts-Bestrebungen weiterbringt, und gegenüber unseren Partnern die- sen auch zur gebührenden Beachtung und Einbezug in die weiteren Entwicklungen in Verwaltung und Politik verhilft. Die Tagung bietet eine ausgezeichnete Plattform, die Teilnehmenden über die vielfältigen bereits laufenden Aktivitäten sowie über geplante Projekte zu informieren. Synergie- und Vernetzungspotential werden aufgezeigt, und unsere Basis bringt wertvolle Anregungen zur Weiterentwicklung der SAQM ein. Die Teilnehmenden wählen schliesslich mit Hé-

\section{Das erste «Forum Qualität» setzt ein starkes Zeichen des Miteinanders.}

lène Beutler (Schweizerische Gesellschaft für Kinder- und Jugendpsychiatrie und -psychotherapie), Jürg Nadig (Schweizerische Gesellschaft für Medizinische Onkologie und Swiss Federation of Specialities in Medicine), Urs Müller (Schweizerische Gesellschaft für Orthopädie und Traumatologie) und Johannes Brühwiler (Hausärzte Schweiz) den Steuerungsausschuss, welcher die Breite der Ärzteschaft abbildet und vertreten kann. Dieses starke Zeichen des Miteinanders prägt auch weiterhin unsere Arbeit in der SAQM. Gemeinsam sammeln wir unsere Erfahrungen und sind offen für Anliegen der Basis wie auch unserer Partner. Dankbar für die erlebte Unterstützung und für die wertvollen Rückmeldungen der Tagungsteilnehmenden gehen wir die nun anstehenden Arbeiten an.

Noch gibt es Fachgesellschaften, kantonale Ärztegesellschaften und anerkannte Dachverbände, welche bisher keinen Delegierten und Ersatz-Delegierten bezeichnet haben, Nach diesem ersten Treffen hoffen wir nun auf die entsprechenden Anmeldungen, um dann am 14. November 2013 anlässlich des nächsten Treffens des «Forum Qualität» der SAQM alle vertreten zu haben. Es ist also noch nicht zu spät, mit an Bord zu kommen!

Dr. med. Christoph Bosshard, Mitglied des Zentralvorstandes der FMH, Verantwortlicher Ressort Daten, Demographie und Qualität 